\title{
CBT at the Crossroads: The Rise of Transdiagnostic Treatments
}

\author{
Carmen Schaeuffele ${ }^{1}$ (D) Ava Schulz ${ }^{2}$. Christine Knaevelsrud ${ }^{3}$. \\ Babette Renneberg ${ }^{1} \cdot$ Johanna Boettcher ${ }^{1,4}$
}

Accepted: 27 October 2020 / Published online: 20 November 2020

(C) The Author(s) 2020

\begin{abstract}
Transdiagnostic treatments span a heterogeneous group of interventions that target a wider range of disorders and can be applied to treat several disorders simultaneously. Several meta-analyses have highlighted the evidence base of these novel therapies. However, these meta-analyses adopt different definitions of transdiagnostic treatments, and the growing field of transdiagnostic therapies has become increasingly difficult to grasp. The current narrative review proposes a distinction of "one size fits all" unified and "my size fits me" individualized approaches within transdiagnostic therapies. Unified treatments are applied as "broadband" interventions to a range of disorders without tailoring to the individual, while individualized treatments are tailored to the specific problem presentation of the individual, e.g., by selecting modules within modular treatments. The underlying theoretical foundation and relevant empirical evidence for these different transdiagnostic approaches are examined. Advantages and limitations of the transdiagnostic treatments as well as future developments are discussed.
\end{abstract}

Keywords Transdiagnostic · Unified · Individualized · Tailored · Cognitive behavioral therapy

Carmen Schaeuffele

carmen.schaeuffele@fu-berlin.de

1 Clinical Psychology and Psychotherapy, Freie Universität Berlin, Habelschwerdter Allee 45, 14195 Berlin, Germany

2 Department of Psychiatry, Psychotherapy and Psychosomatics, University of Zurich, Zürich, Switzerland

3 Clinical-Psychological Intervention, Freie Universität Berlin, Berlin, Germany

4 Clinical Psychology and Psychotherapy, Psychologische Hochschule Berlin, Berlin, Germany 
In its early stages, cognitive behavioral therapy (CBT) comprised a collection of general techniques like cognitive restructuring, which were applied to several disorders (Beck and Haigh 2014). Over the years, research advanced and identified an increasing number of characteristics of specific disorders, followed by increasingly narrow definitions of diagnoses (American Psychiatric Association 1980, 2000, 2013). This disorder-specific approach was successful: Effective disorderspecific treatments were developed and resulted in a rise of evidence-based treatments for specific diagnoses (see Hofmann et al. 2012 for a review of metaanalyses).

The focus on disorder-specifics is, however, in stark contrast to high comorbidity between disorders with $40 \%$ of patients reporting more than one diagnosis (Jacobi et al. 2014). High comorbidity rates are likely not a simple co-occurrence of disorders but a result of a shared common underlying basis between disorders, as increasing evidence suggests (e.g., Caspi et al. 2014; Lahey et al. 2017a, 2017b). Instead of unique processes responsible for the onset and maintenance of single disorders, shared transdiagnostic processes that are causally interrelated with several mental disorders have been identified (see Harvey et al. (2004) and Mansell et al. (2008) for an overview). These findings are also reflected in the development of alternative classification systems like RDoC (Insel et al. 2010) or HiTOP (Kotov et al. 2017) that go beyond categorical diagnoses. In their initial review, Harvey et al. (2004) identified 12 definite transdiagnostic processes across several domains, like attentional biases, recurrent negative thinking, and avoidance behavior. Several other transdiagnostic processes have been proposed since then, such as perfectionism (Egan et al. 2011) and intolerance of uncertainty (Carleton 2016; Mahoney and McEvoy 2012; McEvoy and Erceg-Hurn 2016; Shihata et al. 2016). Transdiagnostic processes differ in how broad or narrow they are. For example, maladaptive emotion regulation (Aldao et al. 2010, 2016; Sloan et al. 2017), experiential avoidance (Hayes et al. 1996), and neuroticism (Barlow et al. 2013; Shackman et al. 2016) encompass many of the narrower processes described, like avoidance behaviors and thought suppression. The broader processes could also be viewed as higher order processes or underlying constructs that are expressed in different specific processes.

Other authors have proposed a different perspective on the link between mechanisms and the development and maintenance of psychopathology: Morris and Mansell (2018) have suggested that it might be the rigid and inflexible application of processes rather than the processes themselves that links processes and psychopathology. On the other hand, Dalgleish et al. (2020) emphasize that greater consideration needs to be given to the mental content of processes - after all, the content of a patient's beliefs and thoughts is central in therapy.

As of yet, there is no comprehensive theoretical framework explaining the distinct relation, interplay, or hierarchical organization of transdiagnostic processes (Aldao and Nolen-Hoeksema 2010; Hong and Cheung 2015; Mansell and McEvoy 2017). The research on transdiagnostic processes is still in flux and future studies that are stimulated and informed by alternative classification systems like RDoC and HiTOP will likely help to further clarify and shed light on transdiagnostic processes. A framework of transdiagnostic processes that integrates a hierarchical organization, the degree of impairment, as well as the mental content of processes 
might prove to be beneficial and informative for diagnostic assessment and therapy planning.

This shift from the emphasis of differences between disorders to the commonalities and shared processes is also evident in the development of novel transdiagnostic treatments. Transdiagnostic treatments, broadly speaking, aim to address several comorbidities simultaneously (Clark and Taylor 2009). While there is no uniform definition of transdiagnostic treatments, they have been defined as treatments that "apply the same underlying treatment principles across mental disorders, without tailoring the protocol to specific diagnoses" (McEvoy et al. 2009 , p. 21) or "are made available to individuals with a wide range of diagnosis, and that do not rely on knowledge of these diagnoses to operate effectively" (Mansell et al. 2009, p. 9). Many of the newly developed transdiagnostic interventions explicitly target shared transdiagnostic processes in therapy in order to facilitate change across several diagnoses. In Table 1, we provide an overview of these interventions and their targeted mechanism. By targeting shared processes and several diagnoses at once, transdiagnostic treatments have several advantages. The transdiagnostic approach might (a) reduce overall treatment length and cost, (b) enhance clinical practicability and simplify clinical training, (c) bridge the gap between research and practice and facilitate the dissemination of evidence-based treatments, and (d) help therapists to factor in all processes important for therapeutic progress by widening their view beyond the diagnosis (Barlow et al. 2016; Leichsenring and Steinert 2018; Sauer-Zavala et al. 2017; Smith and Grawe 2005).

Whether the transdiagnostic approach achieves these results and is advantageous in comparison to disorder-specific approaches are empirical questions. Reviews and meta-analyses show strong efficacy for these types of novel treatments across treatment settings and delivery formats (e.g. Fusar-Poli et al. 2019; Newby et al. 2015; Păsărelu et al. 2017; Pearl and Norton 2017; Reinholt and Krogh 2014). These reviews apply different definitions of transdiagnostic approaches and focus on a subset of transdiagnostic treatments in depth. They focus, for example, on the evidence for transdiagnostic treatment versus disorder-specific treatments in anxiety disorders (Pearl and Norton 2017), solely on internet-based delivery of transdiagnostic treatments (Newby et al. 2016; Păsărelu et al. 2017) or review the evidence for one specific transdiagnostic protocol (Cassiello-Robbins et al. 2020; Sakiris and Berle 2019). The current narrative review will adopt a wider definition of transdiagnostic treatments. We define transdiagnostic treatments broadly as treatments that target several comorbidities at once, either by providing a protocol that targets shared mechanisms between disorders or by individualizing a treatment to the specific person and by that targeting the unique combination of comorbidities. This a more comprehensive definition than older definitions of transdiagnostic treatments that do not include tailored treatments (Mansell et al. 2009; McEvoy et al. 2009). This definition is reflective of the increase in modularization and individualization of transdiagnostic treatment protocols in recent years. Beyond an investigation of the empirical evidence, we will specifically highlight innovative developments in both face-to-face as well as internet-delivered settings that have benefits for increasing effectiveness and practicability of treatments. We will discuss the underlying theoretical foundation, as well as the advantages and limitations in their treatment of mental disorders. 
Table 1 Transdiagnostic interventions that specifically target shared mechanisms between disorders

\begin{tabular}{|c|c|c|}
\hline Therapy & Transdiagnostic process & $\begin{array}{l}\text { Potential higher } \\
\text { order mechanism }\end{array}$ \\
\hline
\end{tabular}

Rumination-focused therapy (Watkins et al. 2007, 2011)

Worry and sadness program (Newby et al. 2013)

Metacognitive therapy (Wells 2011)

Attentional bias modification (MacLeod and Mathews 2012)

Behavioral activation (Martell et al. 2013)

Transdiagnostic behavior therapy (Gros 2014)

Acceptance and commitment therapy (Hayes et al. 1999)

False safety behavior elimination therapy (Schmidt et al. 2012)

CBT for clinical perfectionism (Riley et al. 2007; Shafran et al. 2004)

Treatment model by Einstein (2014)
Repetitive negative thinking/rumination
(Ehring and Watkins 2008;
Harvey et al. 2004)

Metacognitive beliefs (Harvey et al. 2004)

Attentional bias (Harvey et al. 2004)

Avoidance behavior (Harvey et al. 2004)

Experiential avoidance (Harvey et al. 2004)

Safety behavior (Harvey et al. 2004)

Perfectionism (Egan et al. 2011)

Intolerance of uncertainty (Carleton 2016; Einstein 2014; Mahoney and McEvoy 2012; McEvoy and Erceg-Hurn 2016; Shihata et al. 2016)

Dialectic behavioral therapy/skills training (Linehan 1993a, 1993b)

Affect regulation training (Berking and Lukas 2015)

Emotion regulation training (Mennin and Fresco 2014; Renna et al. 2017)

Unified Protocol

(Barlow 2011; Ellard et al. 2010)
Emotion regulation (Aldao et al. 2010, 2016; *

Berking and Wupperman 2012;

Sloan et al. 2017)

Negative affect/neuroticism

(Barlow et al. 2013; Shackman et al. 2016)

Several studies have begun to investigate the interplay and underlying structure of transdiagnostic processes using factorial analyses (e.g., Bird et al. 2013; Mansell and McEvoy 2017; Naragon-Gainey et al. 2017; Naragon-Gainey and Watson 2018). While there is emerging evidence that one core process is underlying several transdiagnostic processes, this core process has not been definitively labeled which is also hindered by different selections of questionnaires between the studies

\section{Classifications of Transdiagnostic Treatments}

With the emergence of new transdiagnostic treatments and a growing interest in researching transdiagnostic processes, the field of transdiagnostic treatments becomes increasingly difficult to grasp. Many approaches hold an intrinsically transdiagnostic stance towards psychopathology, like psychodynamic approaches, or can be applied transdiagnostically, like many of the "third wave approaches" within CBT. Other approaches have been specifically developed with a transdiagnostic perspective in mind and transdiagnostic applicability is their distinguishing trademark. When transdiagnostic treatment protocols were first developed, the majority represented unified comprehensive 
protocols that were applied to several diagnoses. Since then, alternatives to unified transdiagnostic approaches have been proposed, in the form of more individualized, tailored, or modular interventions. The field of transdiagnostic treatments is thus diverse and heterogeneous. Transdiagnostic treatments span a spectrum of treatments. They are either applied as a "broadband" intervention to a range of disorders without tailoring to the individual or are individualized and tailored to the specific problem presentation of the individual. To help differentiate between the various transdiagnostic approaches, we propose a distinction between unified "one size fits all" and individualized "my size fits me" transdiagnostic approaches, as depicted in Fig. 1. Distinguishing between unified and individualized treatments within transdiagnostic treatments can be useful for establishing the differential evidence of approaches. This differentiation can also help to determine to which degree individualization enhances treatment outcomes. In addition, previous reviews of transdiagnostic treatments have often excluded individualized approaches as these are less clearly labeled as transdiagnostic treatments. In our understanding, unified transdiagnostic approaches refer to protocols that, as a whole, are applied to a range of disorders and have been developed in recent years with a transdiagnostic focus. These treatments often target hypothesized shared mechanisms across disorders (coined "shared mechanisms treatments" by Sauer-Zavala et al. (2017) and subsumed under the "universal interventions" by Dalgleish et al. (2020)). On the other hand, individualized transdiagnostic approaches encompass a range of approaches where a treatment is tailored to the individual. In this context, "tailoring" can mean different things, e.g., selecting an individual sequence and modules in modular treatments (Chorpita and Weisz 2009; Johansson et al. 2012; Murray et al. 2014; Silfvernagel et al. 2012), adding disorderspecific information to the same sequence of modules (Berger et al. 2014), or combining elements from evidence-based treatments for an individual treatment plan (Grawe 2004; Persons 2012). Naturally, what is true for both unified and individualized treatments is that they differ in the "breadth of application" (Dalgleish et al. 2020). They range from narrow applications within classes of disorders like eating disorders (Fairburn et al. 2003), or anxiety disorders (Norton and Hope 2005), to applications for higher-order spectra like the internalizing disorders (Barlow 2011) to no restriction in application (Grawe 2004; Persons 2012). Transdiagnostic treatments also differ in delivery mode (face-to-face or internet-based) or target population. While most of these treatments are tailored towards adults, some are applied to children and adolescents (Chorpita and Weisz 2009; Ehrenreich-May et al. 2017).

In the next section, we will briefly highlight third-wave therapy approaches as well as developments outside the CBT field under the transdiagnostic spotlight, before elaborating on unified "one size fits all" approaches versus individualized "my size fits me" approaches.

\section{Third-Wave Therapies as Transdiagnostic Treatments and Transdiagnostic Developments Outside CBT}

\section{Third-Wave Therapies as Transdiagnostic Treatments}

A first shift from the disorder-specific focus within CBT can be observed in the emergence of "third wave therapies" in the 1990s. These therapies have been developed 


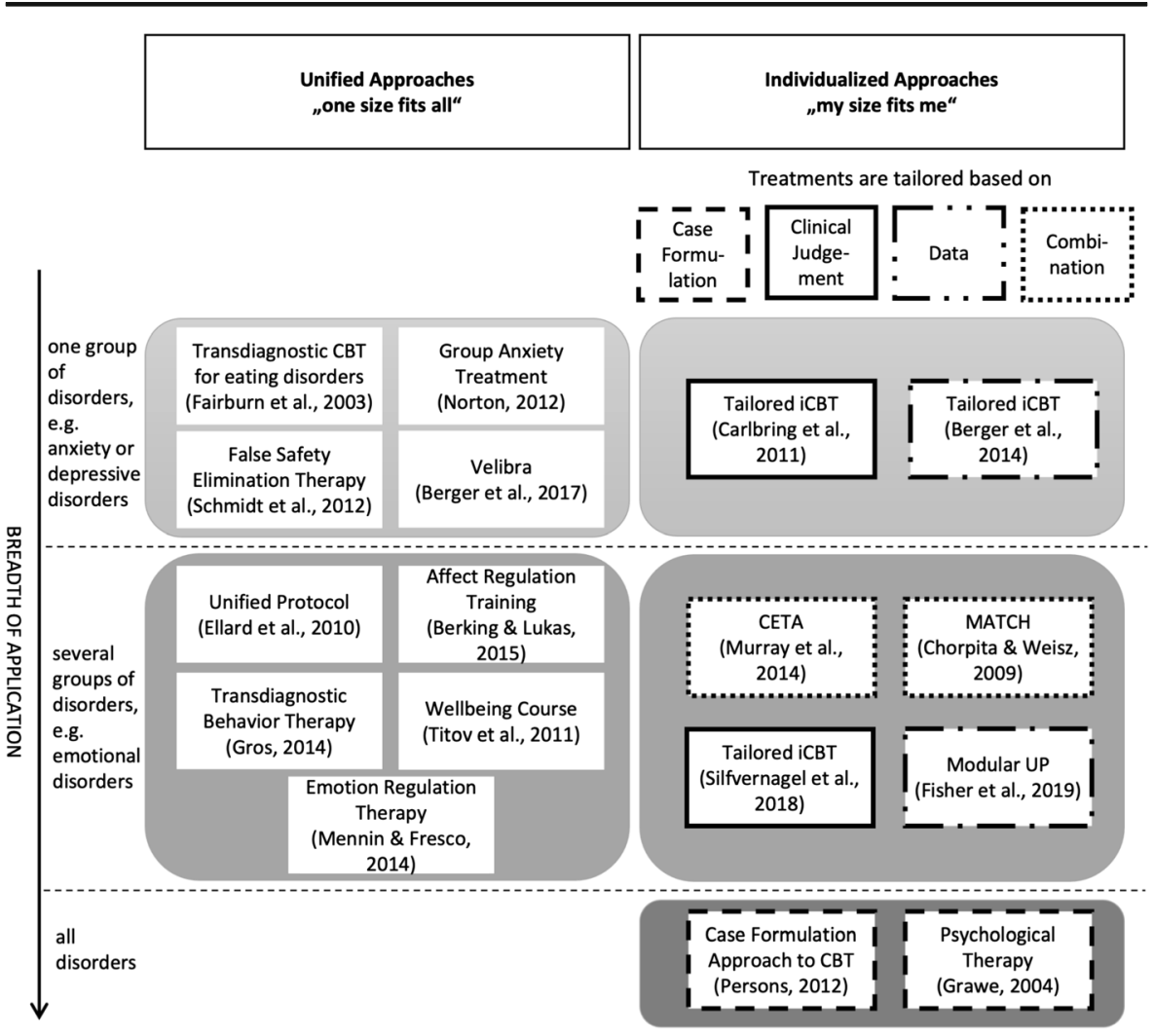

Fig. 1 Proposed classification of transdiagnostic therapies

with different populations in mind and are based on different theoretical assumptions. They share, however, an emphasis on how one manages, observes, and processes experiences and promote a mindful and accepting approach towards these experiences. Thus, some of the third-wave therapies are compatible with the transdiagnostic perspective and recent publications have highlighted the transdiagnostic applicability of these treatments (see below).

\section{Metacognitive Therapy (MCT)}

MCT is a transdiagnostic therapy approach that focuses on metacognitionscognitions about uncontrollability, dangerousness, or importance of thoughts and feelings (Wells 2011). Instead of changing the content of a specific thought, MCT targets metacognitions that maintain repetitive negative thinking by different techniques like mindfulness exercises or behavioral experiments. A metaanalysis aggregated results across 19 studies and found that MCT is effective for the treatment of various disorders: MCT was found to be equally as effective as CBT for treating depression and superior to CBT in treating anxiety (Philipp et al. 2019). 


\section{Acceptance and Commitment Therapy (ACT)}

ACT is an acceptance-based approach that aims at increasing psychological flexibility by targeting several processes that the developers theorize to be relevant for change: acceptance, cognitive defusion, self as context, values, and committed action (Hayes et al. 1999, 2006). While earlier meta-analyses questioned the evidence-base for ACT (Ost 2008, 2014), newer meta-analyses have strengthened ACT's evidence base for treating anxiety disorders, depression, addiction, and somatic health problems with similar outcomes as established psychological interventions (A-Tjak et al. 2015; Bai et al. 2020; Coto-Lesmes et al. 2020; Hacker et al. 2016). The flexibility in which ACT principles are applied to various disorders highlights the transdiagnostic nature of ACT (Dindo et al. 2017).

\section{Mindfulness-Based Interventions}

Mindfulness-based interventions are another form of immensely popular transdiagnostic treatments. Mindfulness-based interventions subsume many different forms of interventions, ranging from stand-alone internet-based applications (Boettcher et al. 2014) to mindfulness-based stress reduction (Kabat-Zinn 2003) or mindfulnessbased cognitive therapy (Segal et al. 2002). While some of the mindfulness interventions have been developed for specific application - such as mindfulness-based cognitive therapy to prevent relapse in recurrent depressive disorders - mindfulness interventions generally have been applied to various populations and led to reductions in symptoms like anxiety or depression and thus can be considered transdiagnostic (Goldberg et al. 2018).

\section{Dialectic Behavioral Therapy (DBT)}

DBT's focus has been on the treatment of borderline personality disorder for which its efficacy has been demonstrated in meta-analysis (Panos et al. 2014). One of the DBT goals is to advance emotion regulation - a process that is now recognized across a multitude of disorders (e.g. Aldao et al. 2016). Consequently, DBT's potential as a transdiagnostic treatment has been emphasized (Lungu and Linehan 2016; Ritschel et al. 2015). Skills training, as one of the central treatment components to enhance emotion regulation, has been applied as a stand-alone intervention to various populations and has shown promising results (Neacsiu et al. 2014). While elements of DBT have merit for transdiagnostic applications, the full rationale including its focus on the treatment of suicidal thoughts and self-injury is possibly not universally fitting for a wide range of individuals.

\section{Developments Outside of CBT}

In contrast to CBT, the conceptualization of a patient's presentation in psychodynamic approaches has traditionally been less focused on the diagnostic category and more on structure, conflicts, and defense mechanisms. Opposite to the discussion within the field of CBT, there are some calls in the field of psychodynamic approaches for more disorder-specific tailoring because psychodynamic treatments may be too universal to 
fulfill their full potential (Leichsenring and Steinert 2018). Moving from a universal approach in the direction of more disorder-specific applications, manualized psychodynamic transdiagnostic unified treatments that target a group of disorders have been proposed. These unified psychodynamic protocols integrate components that have been shown to be efficacious in randomized controlled trials (RCTs) for specific groups of disorders, e.g., for social anxiety and generalized anxiety disorders (Leichsenring et al. 2009, 2013). Unified psychodynamic treatments have been proposed for anxiety disorders (Leichsenring and Salzer 2014), depressive disorders (Leichsenring and Schauenburg 2014), and emotional disorders (Leichsenring and Steinert 2018). Since the treatment protocols are currently still under investigation, there is no empirical evidence of their transdiagnostic effect as of yet.

Besides psychodynamic approaches, experiential approaches like emotion-focused therapy have also put forward a transdiagnostic application of their rationale (Timulak and Keogh 2020). The protocol combines targeting shared mechanisms between disorders with a modular selection based on symptoms the patients presents with in order to help the patient to deal with painful emotions and unmet needs (Timulak and Keogh 2020).

Another approach outside of CBT that can be considered transdiagnostic is systemic therapy that does not use diagnoses as a determining ground for treatment. Instead, emphasis is placed on how an individual's relationships contribute to the development and maintenance of psychological symptoms (Stratton 2016). Systemic therapy aims at changing these conditions in a brief, resource-oriented, and solution-focused manner (Stratton 2016) and has been shown to be effective for a range of mental disorders in adolescents and adults (Carr et al. 2020; Pinquart et al. 2016; Riedinger et al. 2017).

\section{Summary of Third-Wave Therapies Within CBT and Developments Outside of CBT}

Third-wave therapies within CBT as well as psychodynamic and systemic approaches have implemented transdiagnostic principles and can thus be considered efficacious transdiagnostic treatment options. While some CBT third-wave therapies were originally developed for specific disorders, they have been extended to the treatment of other disorders. Therein lies an important difference to transdiagnostic CBT: it may make a difference to extend a treatment to other diagnoses than to develop one on a theoretical and empirical basis with many targeted diagnoses in mind. When considering the transdiagnostic applicability of a certain protocol, one should not only consider studies on symptom improvement but what should also be taken into account are patient satisfaction, adherence, and amelioration of comorbid complaints. What becomes apparent is that third-wave and transdiagnostic therapies within CBT are moving in the direction of psychodynamic traditions by increasingly focusing on general underlying mechanisms. The development in both schools, CBT, and psychodynamic approaches, from general techniques to narrower disorder-specific or transdiagnostic protocols reflects the question of how universal a therapy should be and if and how accounting for disorder-specifics is important to deliver effective therapy. 


\section{Unified Treatments: “One Size Fits All”}

Since the mid-2000s, CBT treatments have been developed with a transdiagnostic perspective in mind, targeting shared mechanisms between disorders and thus offering a one size fits all approach to a wide range of disorders. The following are examples of the most investigated transdiagnostic interventions to highlight their advantages and areas of use.

\section{Unified Protocol}

One of the most researched examples of this "one size fits all approach" is the Unified Treatment Protocol for Emotional Disorders (UP) (Ellard et al. 2010). This CBT protocol is based on the assumption that an increased negative affect and attempts to avoid these emotions are at the core of emotional disorders. The UP incorporates three core principles to target commonalities between emotional disorders: (a) increasing mindful awareness of emotions, (b) increasing cognitive flexibility, and (c) reducing emotion avoidance. Originally developed for an individual treatment setting, modifications were made for the application in group settings (e.g. Maia et al. 2013; Reinholt et al. 2017), internet-based formats (Tulbure et al. 2018), specific subgroups (such as patients after a suicide attempt (Bentley et al. 2017)), and children and adolescents (Ehrenreich-May et al. 2017). A meta-analysis of studies investigating the UP revealed large effect sizes regarding the reduction of anxiety and depression (Sakiris and Berle 2019).

\section{Transdiagnostic CBT for Eating Disorders}

While most of the protocols described thus far focus on anxiety and depressive disorders, another line of research argues for a transdiagnostic approach for eating disorders. Fairburn et al. (2003) argue that bulimia, anorexia, and a large group of atypical eating disorders share four maintaining processes: perfectionism, low selfesteem, mood intolerance, and interpersonal difficulties. One study compared two kinds of transdiagnostic treatments, one focusing solely on eating disorder psychopathology and one targeting these shared underlying mechanisms, to waitlist (Fairburn et al. 2009). They found both treatments superior to waitlist with similar reductions in symptoms (Fairburn et al. 2009). However, patients with deficits in the four proposed mechanisms benefited more from the complex than the simpler transdiagnostic treatment (Fairburn et al. 2009). They concluded that the simpler treatment should be the default treatment option and the more complex treatment should be prescribed to patients with marked deficits in these underlying mechanisms.

\section{Group Anxiety Treatment Protocol}

The Anxiety Treatment Protocol by Norton (2012) is the transdiagnostic group treatment with the largest evidence-base (Norton 2012; Norton and Barrera 2012; Norton and Hope 2005). The protocol consists of 12 group sessions and focuses on classical CBT components such as psychoeducation, cognitive restructuring, exposure exercises, and relapse prevention. A second part of the intervention addresses underlying 
perceptions of uncontrollability, unpredictability, and threat that are shared between different anxiety disorders. Here, cognitive techniques are used to challenge these core beliefs. RCTs investigating the efficacy of this protocol revealed large within group effects regarding the reduction of anxiety and depression (Norton 2012; Norton and Hope 2005), as well as non-inferiority when compared to diagnosis-specific group CBT (Norton and Barrera 2012).

Other examples of transdiagnostic unified approaches that target shared mechanisms between disorders include treatments that focus on the enhancement of adaptive emotion regulation like Affect Regulation Training (Berking and Lukas 2015) as well as Emotion Regulation Therapy (Mennin and Fresco 2014; Renna et al. 2017). Examples of treatments that focus on behavioral processes include Transdiagnostic Behavior Therapy for veterans with affective disorders (Gros 2014) and False Safety Behavior Elimination Therapy for anxiety disorders (Riccardi et al. 2017; Schmidt et al. 2012).

\section{Unified Interventions Applied over the Internet}

The dynamic field of computer-assisted or internet-based CBT (iCBT) was among the first to implement transdiagnostic interventions (e.g. Newby et al. 2013; Titov et al. 2010, 2011). Meta-analyses demonstrated that transdiagnostic intervention can be successfully and effectively delivered over the internet (Newby et al. 2016; Păsărelu et al. 2017).

One of the transdiagnostic internet-based interventions that was investigated in a variety of studies is the self-guided intervention velibra (e.g. Berger et al. 2017). Velibra consists of six treatment modules to be completed over the course of 9 weeks and is presented in a simulated dialog, adapted to the clients' responses. Conceptually, it focuses on transdiagnostic principles, such as the evolutionary adaptive function of emotions, experiential avoidance, and approach vs. avoidance motivation. When velibra was combined with care-asusual (which included any type of psychological or psychiatric treatment), the combined treatment has been shown to be more effective in reducing anxiety and depression than care-as-usual by itself (Berger et al. 2017).

The Wellbeing Course by Titov et al. (2011) is one of the most commonly investigated transdiagnostic iCBT protocols. It consists of five sessions to be completed in 8 weeks, focusing on (a) symptom identification and formulation, (b) thought monitoring and challenging, (c) relaxation and pleasant activity scheduling, (d) graded exposure, and (e) relapse prevention. Like the majority of internet-based interventions, the Wellbeing Course is characterized by a didactic teaching method and homework assignments to facilitate skill acquisition. It can be realized with or without clinician guidance, which is provided either via telephone or written messages. A series of studies investigated the Wellbeing Course for patients with different primary diagnoses across the spectrum of anxiety disorders and depression compared to the respective disorder-specific protocols and failed to find significant differences between the two treatment modalities (Dear et al. 2015). 


\section{Summary of Unified Approaches}

The majority of unified approaches targets shared mechanisms between disorders in order to treat several disorders simultaneously. There are also unified transdiagnostic approaches that do not target shared processes between disorders, but instead target non-psychopathological outcomes shared between disorders, e.g.. iCBT for loneliness (Käll et al. 2020). Taken together, there is a strong evidence base for a multitude of unified approaches across settings and delivery modes. Although the empirical evidence is promising and the ease of use of unified treatment protocols is appealing, this approach also has several limitations. For example, the degree of unification naturally leads to a loss of individualization, which can be detrimental to treatment satisfaction and adherence. Unified protocols may also not present the most parsimonious treatment plan: a depressed patient that suffers predominantly from negative thoughts might not need the full treatment package, which also includes techniques like behavioral activation. Another point of critique is that some of the interventions discussed only target a group of disorders, like eating or anxiety disorders, narrowing their applicability. Following these shortcomings, more individualized and modularized transdiagnostic approaches have been put forward to take into account an individual's idiographic problems, not based on diagnostic classification, but based on individual symptom combinations. Some of the treatments discussed have already been applied as modular approaches or have been extended to address further diagnoses and comorbidities (e.g., Barlow and Farchione 2018).

\section{Individualized Approaches: "My Size Fits Me"}

Similar to developments in medicine, researchers within the field of clinical psychology are calling for advancing precision psychotherapy (Insel and Cuthbert 2015). Optimally matching patients to treatments according to certain baseline characteristics has the potential to enhance treatment outcomes (Cohen and DeRubeis 2018; DeRubeis et al. 2014). The goal is to put the individual in focus and provide the most fitting intervention for this specific person. Individualization is currently realized based on different grounds. Tailored transdiagnostic approaches differ in how they select treatment modules or interventions for the individual patient. One classical example of individualization is the use of clinical case formulation to integrate interventions from different treatment packages. Instead of integrating components across treatments, modular treatment packages have been proposed that allow for a flexible adaptation within one treatment package. Decisions on module selection in modular treatments can be based on (a) clinical judgment, factoring in diagnosis or impression in assessment interviews, (b) data, relying on baseline assessment data or more advanced algorithms, or (c) a combination of clinical judgment and data, with approaches differing in how much weight they put on either data or clinician input and in how flexible intervention selection is handled. In the following, we will present different examples of individualized transdiagnostic interventions, ranging from the idiosyncratic caseformulation approach to purely data driven approaches. 


\section{Clinical Case Formulation}

In clinical practice, manualized therapies have been criticized by practitioners. A diagnosis does not inform treatment planning sufficiently, and possibly as a consequence, the majority of treatments delivered in practice are not evidence-based (Layard and Clark 2014). Some of these critics raise valid points with manuals oversimplifying complex problems or ignoring important patient characteristics. Furthermore, most manuals do not fit routine care settings in session spacing and frequency, often either prescribing too many or too few sessions to match mental health care realities. In practice, a frequently used method to tailor therapy to the individual is on the basis of clinical case formulation. While clinical case formulation is not a therapeutic approach per se, it is implemented in many manualized treatment protocols at the beginning of therapy or can be applied as a decision basis for integrating components across different protocols. In the following, we will present two approaches where clinical case formulation is central to the therapeutic approach.

Persons (2012) has developed a framework, "the case formulation approach to CBT", that offers a compromise between tailoring treatments to the individual and delivering evidence-based treatments and by that bridging the gap between science and practice. The case formulation aims at translating the nomothetic (general) protocol into an idiographic (individualized) treatment plan (Persons 2012). Within this framework, the clinician collaboratively formulates hypotheses about the transdiagnostic mechanisms that cause and maintain all of the patient's problems and disorders based on assessment and observation. Based on this, a treatment is designed that specifically targets those hypothesized mechanisms. An important cornerstone of this approach is the monitoring of progress at every session in order to evaluate the treatment's effectiveness and test the hypotheses (Persons 2012). While this approach gives clinicians guidelines for how to deliver individual therapy in a systematic way, clinicians are still confronted with a problem: It is necessary to dissect treatment protocols and pick and choose elements across different protocols. Even if all protocols from which components were chosen are evidence-based the combined treatment might not be (Persons 2016). Clinical case formulation has been seldomly compared to manualized treatments in a systematic way (Eells 2013). Emerging research suggests, however, that tailoring treatments to transdiagnostic patient characteristics, e.g., in terms of cultural adaptation, religion/spirituality or to treatment preference, will enhance treatment effects which could be incorporated in individual case formulation (Norcross and Wampold 2018).

Another possibility lies in tailoring treatments to the motives of the patient. The motive-oriented therapeutic relationship is an important component of Grawe's Psychological Therapy, a case formulation approach developed by Klaus Grawe in the 1990s. Grawe's Psychological Therapy offers a different perspective on delivering effective and individualized therapy by emphasizing mechanisms of change over psychotherapeutic interventions and techniques (Grawe 2004). Based on the clinical case formulation, an individual therapy plan is derived that optimally targets these change mechanisms and is orientated towards the patient's motives (Grawe et al. 1994). The clinical case formulation (plan analysis) itself is captured as a hierarchical depiction of a patient's individual motives and schemas linking the patient's behavior to four 
universal basic psychological needs (for a detailed description of plan analysis see Caspar, 1995 and 2019).

Thus far, the majority of studies on Grawe's Psychological Therapy or motiveoriented relationships are naturalistic or observational in character. In a naturalistic study, two treatments (group and individual) based on plan analytic case formulation were compared to two treatments without plan analytic case formulations (CBT and humanistic treatment) (Grawe et al. 1990). While the treatments only marginally differed in their effectiveness, patients in the individual motiveoriented condition rated their therapist and their experience with therapy more positively and showed improved outcomes at follow-up. Interestingly, therapists in the motive-oriented condition also showed the highest satisfaction rating with their work and the interaction with the patient, they rated their patients as less difficult and were overall more flexible in the techniques used. One RCT compared a 10session brief treatment for borderline personality disorder without and with additional motive-oriented therapeutic relationship focus and found that the motiveorientated condition led to a greater increase in general outcomes and demonstrated positive effects on early changes in self-esteem and alliance (Kramer et al. 2014).

With this ideographic approach, Grawe's Psychological Therapy is not only transdiagnostic but also "transtherapeutical" by bridging different schools and techniques. Grawe's Psychological Therapy provides the clinician with a plausible framework for case formulation and treatment planning and appeals by its openness towards new empiric evidence. Grawe's Psychological Therapy, however, is not widely disseminated outside German-speaking countries and its complex theoretical foundation and encompassing framework requires specific training and supervision. Furthermore, the problem described by Persons (2016) persists. Even though Grawe's Psychological Therapy draws from evidence-based treatments, the effect of the specific combination of interventions chosen for an individual patient is unknown.

Another example of a transdiagnostic case formulation approach with a focus on interpersonal problems is schema therapy (Young 1999) which can be considered a transdiagnostic treatment for various personality disorders (Jacob and Arntz 2013).

\section{Modular Treatments}

In the following, we will present different modular treatments that differ in their approach to module selection.

\section{Module Selection Based on Clinical Judgment}

One approach to select modules is to rely on clinical judgment. Several internetbased interventions use this type of module selection, e.g., by basing the decision on diagnosis and participant preference (Carlbring et al. 2011), impression in the telephone interview (Silfvernagel et al. 2012), or giving online therapists the opportunity to tailor the treatment during treatment (Silfvernagel et al. 2018). These tailored treatment approaches have been compared to waitlist for younger adults and adults with panic attacks (Silfvernagel et al. 2012) and older adults with mixed anxiety and depression (Silfvernagel et al. 2018) and to a moderated discussion 
forum for anxiety disorders (Carlbring et al. 2011) with medium to large effects. These results suggest that tailoring treatments based on clinical judgment produces promising effects. However, they do not answer how clinical judgment compares to other forms of tailoring.

\section{Module Selection Based on Data-Driven Decisions}

Decisions on individualizing content can also be based on data, either on cut-offs in questionnaires or more advanced algorithms.

One study that investigated a tailored internet-based intervention used cutoffs in questionnaire as a basis for tailoring (Berger et al. 2014). They compared tailored to standardized treatments for mixed anxiety disorders (Berger et al. 2014) and found no significant differences between the two conditions, albeit the study was possibly not sufficiently powered to detect differences between treatments. Beyond efficacy, these results raise interesting points in regard to patient-treatment-fit, suggesting that the match between this program and individual problems and goals was higher for participants in the tailored condition than in the standardized condition (Berger et al. 2014).

A more complex algorithm was developed for selecting the best modules for the specific individual's presentation in a modular face-to-face application of the Unified Protocol (Fernandez et al. 2017; Fisher and Boswell 2016). The developed algorithm is based on intensive repeated measurements prior to therapy. Prior to therapy, patients rate symptoms and behaviors several times a day on 30 consecutive days. These data are analyzed regarding individual symptom structure (person-specific factor analyses) and relation of symptoms over time (dynamic factor models) and matched to treatment modules. This concept was investigated in an open trial (Fisher et al. 2019). The authors applied the Unified Protocol as a modular intervention and individualized both module selection and sequence based on either the fully automated algorithm or expert panel selection. The expert panel also based their selection on the person-specific factor analyses and individual dynamic models. Both groups yielded large pre-to post-changes, but the expert panel led to a significantly greater reduction in symptoms than the algorithm in the completer data. They concluded that "although the expectation was that [the algorithm] would sharpen the estimation of symptom hierarchies by not omitting small but crucial details or overemphasizing semantically salient features, it may be that, instead, greater efficacy is derived from a gestalt perspective that better summarizes the nature of the latent variable" (Fisher et al. 2019, p.24). While this strengthens the view that clinical decision-making based on data is superior to purely data-driven decision-making, this type of research is still in its infancy and needs to be investigated further.

\section{Module Selection Based on a Combination of Data and Clinical Judgment}

A combination of data from questionnaires and clinical judgment for module selection has been applied in internet-based as well as face-to-face modular treatments.

Tailored Internet-Based Interventions Johansson et al. (2012) compared tailored with standardized treatment as well as an active control group for mixed depressive 
disorders. Tailoring was decided by discussion in the research group based on diagnoses and self-report questionnaires (Johansson et al. 2012). The tailored treatment yielded large within effect sizes (Johansson et al. 2012). While no difference between standardized and tailored was observed, the tailored condition worked better for participants with more symptom burden and higher comorbidity (Johansson et al. 2012).

Modular Face-to-Face Approaches Several modular face-to-face approaches use a combination of data and clinical judgment for module selection.

Modular Approach to Therapy for Children (MATCH) One of the first modular treatments ever developed was for children and adolescents exhibiting a wide range of problems including anxiety, depression, trauma, and conduct problems (Chorpita and Weisz 2009). MATCH provides a total of 33 corresponding modules for selection. It utilizes a guiding algorithm to select the modules, but the treatment plan can be flexibly adapted if the weekly outcome monitoring indicates poor response. The algorithm aggregated data from several sources, including outcome data as well as data gathered from the youth, their family, and other sources. Based on the data, the treatment team collaboratively decides on adaptation in a weekly review (Chorpita et al. 2017). Two RCTs have compared MATCH to routine community implementation of multiple evidence-based practices (Chorpita et al. 2017), and evidence-based treatments and usual care (Weisz et al. 2012). They found MATCH to be superior in comparison (Chorpita et al. 2017; Weisz et al. 2012) and results indicated that MATCH requires significantly fewer treatment sessions (Chorpita et al. 2017).

Common-Elements-Treatment-Approach (CETA) CETA (Murray et al. 2014) is a tailored intervention addressing symptoms of depressive and anxiety disorders, PTSD, and substance abuse. CETA consists of nine modules, ranging from cognitive restructuring to imagery exposure to problem solving. Module selection is based on data from a questionnaire, covering symptoms of all targeted disorders, as well as suicidal ideation and aggression. Self-report questionnaire data are combined with information from the first session, including the patient's description of the main problems. Based on this information, the therapist and supervisor define a preliminary sequence of modules which can be adapted during therapy, depending on the patient's progress. Initially, CETA was developed to provide evidence-based care in low-income countries. Treatments are conducted by lay counselors who are trained and supervised by CBT therapists. Two RCTs showed very good effects, one in a sample of Burmese refugees (Bolton et al. 2014) and one in survivors of torture and militant attacks in Iraq (Weiss et al. 2015). CETA provides core CBT elements for the most common mental disorders. It aims at parsimony and scalability, thus offering a valid treatment option for populations with limited access to mental health resources.

Shaping Healthy Minds Another modular transdiagnostic intervention is "Shaping Healthy Minds" (Black et al. 2018). Shaping Healthy Minds is a flexible 10-module intervention focused on alleviating symptoms and improving wellbeing for patients with anxiety, unipolar depressive, and stressor-related disorders. The selection and sequence of modules is based on collaborative case formulation prior to treatment 
that takes into account the assessment of core problematic areas of emotional, cognitive, interpersonal, and behavioral processes. Shaping Healthy Minds is currently being investigated in comparison to treatment-as-usual (Black et al. 2018).

\section{Summary of Individualized Approaches}

Individualized approaches encompass a wide range of treatments, from modular face-to-face and internet-based interventions to case-formulation approaches of evidence-based treatments. Overall, the results on individually tailored therapy are promising and suggest that tailored treatments are more effective than control (waitlist, moderated discussion forum and attention control) and can be equally effective as standardized treatment. While the question remains of whether tailored treatments are actually more effective than standardized treatments, they may offer a better match between patient and treatment program (Berger et al. 2014), have advantages in treating high symptom burden/comorbidity (Johansson et al. 2012), lower treatment costs (Nordgren et al. 2012), and require fewer sessions (Chorpita et al. 2017). They may also lead to a higher satisfaction with treatment on the therapist side (Grawe et al. 1990).

\section{Discussion}

Transdiagnostic treatments are an umbrella term for very heterogenous treatments. In this narrative review, we have chosen a broad definition of transdiagnostic treatments, in order to provide a comprehensive overview of this rapid moving field and to extend former reviews that focused primarily on unified approaches. While we hope to offer a broad overview of the emerging transdiagnostic treatments, our review was not systematic and thus does not reflect the whole field of transdiagnostic treatments. Other systematic reviews and meta-analysis, while taking a narrower inclusion of transdiagnostic treatments, offer more depth in this regard. Instead, our aim was to highlight interesting and overlooked developments with merit for further increasing the effectiveness, dissemination, and practicability of transdiagnostic treatments within CBT. Psychodynamic, experiential, and systemic therapeutic approaches have a longstanding tradition of centering different conceptualizations of individual problems beyond a patient's diagnoses. Some of these conceptualizations, for example in regard to common underlying mechanisms between disorders, are shared in transdiagnostic perspectives within the CBT field. Within CBT, transdiagnostic treatments span universal techniques that can be applied across disorders, third-wave therapies, unified treatments, and individualized approaches. Some of the transdiagnostic approaches are comprehensive treatment protocols and some are frameworks on how to conduct therapy transdiagnostically and effectively. Transdiagnostic treatments differ in delivery mode (face-to-face or internet-based) and application. The majority of the transdiagnostic treatments are targeting the internalizing mental disorders: some of them target only a subgroup, like the anxiety (Norton and Hope 2005) or eating disorders (Fairburn et al. 2003), and others include all emotional disorders (Barlow 2011). While this is obvious given that internalizing disorders are highly prevalent and 
share so many commonalities, transdiagnostic treatments that target a wider range and other spectra are a desirable future development. However, unified protocols have limits as to how many different symptoms they can target. After all, extending unified transdiagnostic treatments to address all diagnoses would be contrary to evidence-based therapeutic approaches and neglect knowledge on differences between (groups of) disorders. Furthermore, unified approaches neglect differences between individualsa striking contrast to the trend of more personalization in psychotherapy. The transdiagnostic process perspective - that has gained popularity thanks to the rise of shared mechanism unified treatments-has merit for the further development of treatments and clinical decision tools. A necessary next step is to identify relevant processes across diagnoses and derive therapeutic approaches that target these processes (Hofmann and Hayes 2019; Hopwood et al. 2019). From our point of view, this implementation can only be achieved in individualized approaches.

Transdiagnostic treatments are equally effective to disorder-specific therapy (Pearl and Norton 2017) but it remains unclear at this point if transdiagnostic treatments fulfill their intended purpose of targeting comorbid complaints (González-Robles et al. 2018). The majority of studies included in a recent meta-analysis about the impact of transdiagnostic treatments on comorbidity did not report on the intervention's effect on comorbidity (González-Robles et al. 2018). While transdiagnostic treatments do not seem to be more effective than disorder-specific approaches and their impact on comorbidity is not established yet, they might offer advantages beyond efficacy. Their growing popularity highlights a general need for more clarity, simplicity, and emphasis on commonality. A unified approach might be shorter, more feasible, cost-effective, externally valid, and easier to learn. Transdiagnostic unified treatments have also been portrayed as promising developments to help solve the "too many empirically supported treatments problem" and facilitate the dissemination of evidence-based treatments into clinical practice (Weisz et al. 2014). With so many therapies labeled as transdiagnostic, the differences between them are blurred which might impede the fulfillment of this promise.

So where do we go from here? We envision that the following propositions could move the current state of transdiagnostic treatments further in terms of practicability and effectiveness.

Adopt Alternative Diagnostic Systems The term transdiagnostic still encompasses the word "diagnostic" and implies that treatments should target more than one diagnosis. This implication is challenged with alternative classification systems like HiTOP and RDoC moving beyond the classification of diagnosis and proposing empirically derived systems to classify psychopathology (Insel et al. 2010; Kotov et al. 2017). Embracing these systems might help to advance psychotherapy research and practice. A similar approach to HiTOP could be adopted within psychotherapy research to create an empirically based hierarchical conceptualization of psychotherapy, where techniques of different approaches are integrated and distinguished based on quantitative data (Hopwood et al. 2019). While the development of a hierarchical organization of psychotherapeutic techniques would be a highly complex endeavor, it would have a revolutionary impact on empirical clinical decision-making. 
Embrace Modularization While transdiagnostic treatments adapt to several diagnoses at once, additionally adapting treatments to transdiagnostic patient characteristics may enhance effect sizes and treatment response (e.g. Norcross and Wampold 2018). Individualized approaches that tailor treatments based on clinical case formulations like in Grawe's Psychological Therapy might offer some valuable clinical guidance in this regard. In order to further advance individualization, modular treatments are another prerequisite. Some of the unified treatments discussed, like the Unified Protocol, are already being applied modularly and we will likely see a further increase of modular approaches. However, rules on how to select and order modules are as important as the modular treatment itself. More research is needed on how tailoring should be realized and on what tailoring should be based on: Prior to treatment or dynamic in treatment? Based on clinician-, or algorithm-selection or a combination? In addition, the full potential of technology and outcome monitoring has not yet been utilized in these treatments, e.g., in regard to using machine learning algorithms to flexibly adapt treatment content. Another noteworthy development within tailored internet-based treatments is the self-selection of modules by participants. This approach has been applied in a feasibility trial compared to disorder-specific treatment (Kraepelien et al. 2019) and might represent a future line of research above the more frequently applied methods of module selection.

Match Modules to Specific Processes Until now, the majority of individualized treatments have based treatment selection on patient diagnoses: If patients present with anxiety disorders, they are matched to exposure. If patients present with depression, they are matched to behavioral activation. This approach is understandable as the only evidence base we have is for matching treatments to diagnoses. However, it is, at the same time, overly simplistic given that comorbidity is frequent, and diagnoses are highly heterogeneous within themselves. For example, patients with depressive disorders present with very different problems and symptom clusters (Ballard et al. 2018), which challenges the default prescription of behavioral activation for all depressed patients. Instead, it would be more fruitful to select modules based on processes relevant for the onset and maintenance of the problem, e.g., matching behavioral activation modules to anhedonia or exposure modules to situational avoidance. Hofmann and Hayes (2019) have proposed such an approach and termed it "processbased therapy." However, the authors do not yet provide a convincing rationale of how interventions should best be chosen for the individual patient. Indeed, the claim of linking processes and interventions based on empirical evidence is very difficult to meet. It requires intensive additional research on change processes in psychotherapeutic interventions.

Integrate Technology and Assessments Monitoring the patients' response to treatment and satisfaction with therapy real-time will likely improve the effectiveness of treatment and will especially benefit the outcomes of treatment non-responders (Lambert and Shimokawa 2011). In line with the call for further advancement of personalizing treatments, the incorporation of technology, e.g., in form of technology-assisted assessment, into therapy is imminent. While data-driven approaches, for now, mainly base their tailoring on pre-treatment data, the next steps may include developing "justin-time adaptive interventions" (Wright and Woods 2020). One way to move forward is 
to also integrate internet- and mobile-based treatment modules into face-to-face therapy (blended care) to facilitate assessments and benefit from the flexibility these delivery modes provide (Erbe et al. 2017). Combining patient-rated questionnaire data with clinical impressions and more objective data like adherence to online modules has also proven a fruitful way of preventing treatment failure in internet-based treatments (Forsell et al. 2019). The same data sources could be used to tailor treatment contents and allow for more individualization in transdiagnostic treatments.

Focus on Strengths As Well As Deficits With some exceptions, like Grawe's Psychological Therapy that focuses on patient's strengths as well as weaknesses and emphasizes resource activation as a change mechanism, the majority of transdiagnostic therapies discussed here are deficit orientated. They focus on transdiagnostic processes that are impaired and target these in order to improve symptomatology. Another strategy of personalizing treatments is to "capitalize" on a patient's strengths rather than weaknesses (Cheavens et al. 2012). A single case study investigating the Unified Protocol modules ordered according to strengths versus weaknesses found that capitalizing on patients' strengths led to greater effectiveness and earlier symptom improvement (Sauer-Zavala et al. 2019). While further research is needed, it might be beneficial to also consider identifying strengths in transdiagnostic processes whose capitalization could in turn improve well-being. A similar line of research is to empower the patient by actively involving the patient in the module selection (Kraepelien et al. 2019).

\section{Conclusion}

Transdiagnostic approaches to the conceptualisation and treatment of mental disorders have garnered increased attention in the last decade. Linking back to the origins of CBT, and connecting current evidence highlighting commonalities between mental disorders, transdiagnostic approaches target a wider range of disorders and can effectively treat comorbidities. Thereby, they have the potential to reduce training cost for therapists and increase access to evidence-based treatments for a large number of patients. Modular approaches that can be delivered after a short training period by laymen, like CETA, or internet-delivered interventions especially hold promise in facilitating dissemination and bridging the treatment gap in regions with limited access to mental health care systems. However, the current state of research is limited by small sample sizes, heterogeneous definitions of transdiagnostic approaches, and heterogeneous comparison groups. A promising future approach lies in combining individualization and technology by providing treatments which are based on ideographic problems and strengths, regardless of diagnosis, and are supported by real-time feedback.

Funding Open Access funding enabled and organized by Projekt DEAL.

\section{Compliance with Ethical Standards}

Conflict of Interest The authors declare that they have no conflict of interest. 
Open Access This article is licensed under a Creative Commons Attribution 4.0 International License, which permits use, sharing, adaptation, distribution and reproduction in any medium or format, as long as you give appropriate credit to the original author(s) and the source, provide a link to the Creative Commons licence, and indicate if changes were made. The images or other third party material in this article are included in the article's Creative Commons licence, unless indicated otherwise in a credit line to the material. If material is not included in the article's Creative Commons licence and your intended use is not permitted by statutory regulation or exceeds the permitted use, you will need to obtain permission directly from the copyright holder. To view a copy of this licence, visit http://creativecommons.org/licenses/by/4.0/.

\section{References}

Aldao, A., \& Nolen-Hoeksema, S. (2010). Specificity of cognitive emotion regulation strategies: a transdiagnostic examination. Behaviour Research and Therapy, 48(10), 974-983. https://doi.org/10. 1016/j.brat.2010.06.002.

Aldao, A., Nolen-Hoeksema, S., \& Schweizer, S. (2010). Emotion-regulation strategies across psychopathology: a meta-analytic review. Clinical Psychology Review, 30(2), 217-237. https://doi.org/10.1016/j.cpr. 2009.11.004.

Aldao, A., Gee, D. G., De Los Reyes, A., \& Seager, I. (2016). Emotion regulation as a transdiagnostic factor in the development of internalizing and externalizing psychopathology: Current and future directions. Development and Psychopathology, 28, 927-946. https://doi.org/10.1017/S0954579416000638.

American Psychiatric Association. (1980). Diagnostic and statistical manual of mental disorders (3rd ed.). Washington, DC: American Psychiatric Association.

American Psychiatric Association. (2000). Diagnostic and statistical manual of mental disorders (4th ed., text rev.). Washington, DC: American Psychiatric Association.

American Psychiatric Association. (2013). Diagnostic and statistical manual of mental disorders (5th ed.). https://doi.org/10.1176/appi.books.9780890425596.

A-Tjak, J. G. L., Davis, M. L., Morina, N., Powers, M. B., Smits, J. A. J., \& Emmelkamp, P. M. G. (2015). A meta-analysis of the efficacy of acceptance and commitment therapy for clinically relevant mental and physical health problems. Psychotherapy and Psychosomatics, 84(1), 30-36. https://doi.org/10.1159/ 000365764.

Bai, Z., Luo, S., Zhang, L., Wu, S., \& Chi, I. (2020). Acceptance and commitment therapy (ACT) to reduce depression: a systematic review and meta-analysis. Journal of Affective Disorders, 260, 728-737. https:// doi.org/10.1016/j.jad.2019.09.040.

Ballard, E. D., Yarrington, J. S., Farmer, C. A., Lener, M. S., Kadriu, B., Lally, N., Williams, D., MachadoVieira, R., Niciu, M. J., Park, L., \& Zarate, C. A. (2018). Parsing the heterogeneity of depression: an exploratory factor analysis across commonly used depression rating scales. Journal of Affective Disorders, 231, 51-57. https://doi.org/10.1016/j.jad.2018.01.027.

Barlow, D. H. (2011). Unified protocol for transdiagnostic treatment of emotional disorders. Oxford: Oxford University Press.

Barlow, D. H., \& Farchione, T. J. (2018). Applications of the unified protocol for transdiagnostic treatment of emotional disorders. Oxford: Oxford University Press.

Barlow, D. H., Sauer-Zavala, S., Carl, J. R., Bullis, J. R., \& Ellard, K. K. (2013). The nature, diagnosis, and treatment of neuroticism. Clinical Psychological Science: A Journal of the Association for Psychological Science, 2(3), 344-365. https://doi.org/10.1177/2167702613505532.

Barlow, D. H., Allen, L. B., \& Choate, M. L. (2016). Toward a unified treatment for emotional disordersrepublished article. Behavior Therapy, 47(6), 838-853. https://doi.org/10.1016/j.beth.2016.11.005.

Beck, A. T., \& Haigh, E. A. P. (2014). Advances in cognitive theory and therapy: the generic cognitive model. Annual Review of Clinical Psychology, 10(1), 1-24. https://doi.org/10.1146/annurev-clinpsy-032813153734.

Bentley, K. H., Sauer-Zavala, S., Cassiello-Robbins, C. F., Conklin, L. R., Vento, S., \& Homer, D. (2017). Treating suicidal thoughts and behaviors within an emotional disorders framework: acceptability and feasibility of the unified protocol in an inpatient setting. Behavior Modification, 41(4), 529-557. https:// doi.org/10.1177/0145445516689661. 
Berger, T., Boettcher, J., \& Caspar, F. (2014). Internet-based guided self-help for several anxiety disorders: a randomized controlled trial comparing a tailored with a standardized disorder-specific approach. Psychotherapy (Chicago, Ill.), 51(2), 207-219. https://doi.org/10.1037/a0032527.

Berger, T., Urech, A., Krieger, T., Stolz, T., Schulz, A., Vincent, A., Moser, C. T., Moritz, S., \& Meyer, B. (2017). Effects of a transdiagnostic unguided internet intervention ('velibra') for anxiety disorders in primary care: results of a randomized controlled trial. Psychological Medicine, 47(1), 67-80. https://doi. org/10.1017/S0033291716002270.

Berking, M., \& Lukas, C. A. (2015). The affect regulation training (ART): a transdiagnostic approach to the prevention and treatment of mental disorders. Current Opinion in Psychology, 3, 64-69. https://doi.org/ 10.1016/j.copsyc.2015.02.002.

Berking, M., \& Wupperman, P. (2012). Emotion regulation and mental health: recent findings, current challenges, and future directions. Current Opinion in Psychiatry, 25(2), 128-134. https://doi.org/10. 1097/YCO.0b013e3283503669.

Bird, T., Mansell, W., Dickens, C., \& Tai, S. (2013). Is there a core process across depression and anxiety? Cognitive Therapy and Research, 37(2), 307-323. https://doi.org/10.1007/s10608-012-9475-2.

Black, M., Hitchcock, C., Bevan, A., Leary, O., Clarke, J. C., Elliott, R., Watson, P., LaFortune, L., Rae, S., Gilbody, S., Kuyken, W., Johnston, D., Newby, J. M., \& Dalgleish, T. (2018). The HARMONIC trial: study protocol for a randomised controlled feasibility trial of shaping healthy minds - a modular transdiagnostic intervention for mood, stressor-related and anxiety disorders in adults. BMJ Open, 8(8), e024546. https://doi.org/10.1136/bmjopen-2018-024546.

Boettcher, J., Aström, V., Påhlsson, D., Schenström, O., Andersson, G., \& Carlbring, P. (2014). Internet-based mindfulness treatment for anxiety disorders: a randomized controlled trial. Behavior Therapy, 45(2), 241253. https://doi.org/10.1016/j.beth.2013.11.003.

Bolton, P., Lee, C., Haroz, E. E., Murray, L., Dorsey, S., Robinson, C., Ugueto, A. M., \& Bass, J. (2014). A transdiagnostic community-based mental health treatment for comorbid disorders: development and outcomes of a randomized controlled trial among Burmese refugees in Thailand. PLoS Medicine, 11(11), e1001757. https://doi.org/10.1371/journal.pmed.1001757.

Carlbring, P., Maurin, L., Törngren, C., Linna, E., Eriksson, T., Sparthan, E., Strååt, M., Marquez von Hage, C., Bergman-Nordgren, L., \& Andersson, G. (2011). Individually-tailored, internet-based treatment for anxiety disorders: a randomized controlled trial. Behaviour Research and Therapy, 49(1), 18-24. https:// doi.org/10.1016/j.brat.2010.10.002.

Carleton, R. N. (2016). Into the unknown: a review and synthesis of contemporary models involving uncertainty. Journal of Anxiety Disorders, 39, 30-43. https://doi.org/10.1016/j.janxdis.2016.02.007.

Carr, A., Pinquart, M., \& Haun, M. W. (2020). Research-informed practice of systemic therapy. In M. Ochs, M. Borcsa, \& J. Schweitzer (Eds.), Systemic research in individual, couple, and family therapy and counseling (pp. 319-347). Berlin: Springer International Publishing. https://doi.org/10.1007/978-3-03036560-8_18.

Caspar, F. (1995). Plan analysis: toward optimizing psychotherapy. Seattle, WA: Hogrefe \& Huber Publishers.

Caspar, F. (2019). Plan analysis and the motive-oriented therapeutic relationship. In U. Kramer (Ed.), Case formulation for personality disorders (pp. 265-290). Elsevier. https://doi.org/10.1016/B978-0-12813521-1.00014-X.

Caspi, A., Houts, R. M., Belsky, D. W., Goldman-Mellor, S. J., Harrington, H., Israel, S., Meier, M. H., Ramrakha, S., Shalev, I., Poulton, R., \& Moffitt, T. E. (2014). The p factor: one general psychopathology factor in the structure of psychiatric disorders? Clinical Psychological Science: A Journal of the Association for Psychological Science, 2(2), 119-137. https://doi.org/10.1177/2167702613497473.

Cassiello-Robbins, C., Southward, M. W., Tirpak, J. W., \& Sauer-Zavala, S. (2020). A systematic review of unified protocol applications with adult populations: facilitating widespread dissemination via adaptability. Clinical Psychology Review, 78, 101852. https://doi.org/10.1016/j.cpr.2020.101852.

Cheavens, J. S., Strunk, D. R., Lazarus, S. A., \& Goldstein, L. A. (2012). The compensation and capitalization models: a test of two approaches to individualizing the treatment of depression. Behaviour Research and Therapy, 50(11), 699-706. https://doi.org/10.1016/j.brat.2012.08.002.

Chorpita, B. F., \& Weisz, J. R. (2009). Match-ADTC: Modular approach to therapy for children with anxiety, depression, trauma, or conduct problems. Satellite Beach, FL: PracticeWise.

Chorpita, B. F., Daleiden, E. L., Park, A. L., Ward, A. M., Levy, M. C., Cromley, T., Chiu, A. W., Letamendi, A. M., Tsai, K. H., \& Krull, J. L. (2017). Child STEPs in California: a cluster randomized effectiveness trial comparing modular treatment with community implemented treatment for youth with anxiety, depression, conduct problems, or traumatic stress. Journal of Consulting and Clinical Psychology, 85(1), 13-25. https://doi.org/10.1037/ccp0000133. 
Clark, D. A., \& Taylor, S. (2009). The transdiagnostic perspective on cognitive-behavioral therapy for anxiety and depression: new wine for old wineskins? Journal of Cognitive Psychotherapy, 23(1), 60-66. https:// doi.org/10.1891/0889-8391.23.1.60.

Cohen, Z. D., \& DeRubeis, R. J. (2018). Treatment selection in depression. Annual Review of Clinical Psychology, 14, 209-236. https://doi.org/10.1146/annurev-clinpsy-050817-084746.

Coto-Lesmes, R., Fernández-Rodríguez, C., \& González-Fernández, S. (2020). Acceptance and commitment therapy in group format for anxiety and depression. A systematic review. Journal of Affective Disorders, 263, 107-120. https://doi.org/10.1016/j.jad.2019.11.154.

Dalgleish, T., Black, M., Johnston, D., \& Bevan, A. (2020). Transdiagnostic approaches to mental health problems: current status and future directions. Journal of Consulting and Clinical Psychology, 88(3), 179-195. https://doi.org/10.1037/ccp0000482.

Dear, B. F., Staples, L. G., Terides, M. D., Karin, E., Zou, J., Johnston, L., Gandy, M., Fogliati, V. J., Wootton, B. M., McEvoy, P. M., \& Titov, N. (2015). Transdiagnostic versus disorder-specific and clinician-guided versus self-guided internet-delivered treatment for generalized anxiety disorder and comorbid disorders: a randomized controlled trial. Journal of Anxiety Disorders, 36, 63-77. https://doi. org/10.1016/j.janxdis.2015.09.003.

DeRubeis, R. J., Cohen, Z. D., Forand, N. R., Fournier, J. C., Gelfand, L. A., \& Lorenzo-Luaces, L. (2014). The personalized advantage index: translating research on prediction into individualized treatment recommendations. A demonstration. PLoS ONE, 9(1), e83875. https://doi.org/10.1371/journal.pone. 0083875 .

Dindo, L., van Liew, J. R., \& Arch, J. J. (2017). Acceptance and commitment therapy: a transdiagnostic behavioral intervention for mental health and medical conditions. Neurotherapeutics, 14(3), 546-553. https://doi.org/10.1007/s13311-017-0521-3.

Eells, T. D. (2013). The case formulation approach to psychotherapy research revisited. Pragmatic Case Studies in Psychotherapy, 9(4), 426-447. https://doi.org/10.14713/pcsp.v9i4.1834.

Egan, S. J., Wade, T. D., \& Shafran, R. (2011). Perfectionism as a transdiagnostic process: a clinical review. Clinical Psychology Review, 31(2), 203-212. https://doi.org/10.1016/j.cpr.2010.04.009.

Ehrenreich-May, J., Rosenfield, D., Queen, A. H., Kennedy, S. M., Remmes, C. S., \& Barlow, D. H. (2017). An initial waitlist-controlled trial of the unified protocol for the treatment of emotional disorders in adolescents. Journal of Anxiety Disorders, 46, 46-55. https://doi.org/10.1016/j.janxdis.2016.10.006.

Ehring, T., \& Watkins, E. R. (2008). Repetitive negative thinking as a transdiagnostic process. International Journal of Cognitive Therapy, 1(3), 192-205.

Einstein, D. A. (2014). Extension of the transdiagnostic model to focus on intolerance of uncertainty: a review of the literature and implications for treatment. Clinical Psychology: Science and Practice, 21(3), 280300. https://doi.org/10.1111/cpsp.12077.

Ellard, K. K., Fairholme, C. P., Boisseau, C. L., Farchione, T. J., \& Barlow, D. H. (2010). Unified protocol for the transdiagnostic treatment of emotional disorders: protocol development and initial outcome data. Cognitive and Behavioral Practice, 1, 88-101. https://doi.org/10.1016/j.cbpra.2009.06.002.

Erbe, D., Eichert, H.-C., Riper, H., \& Ebert, D. D. (2017). Blending face-to-face and internet-based interventions for the treatment of mental disorders in adults: systematic review. Journal of Medical Internet Research, 19(9), e306. https://doi.org/10.2196/jmir.6588.

Fairburn, C. G., Cooper, Z., \& Shafran, R. (2003). Cognitive behaviour therapy for eating disorders: a "transdiagnostic" theory and treatment. Behaviour Research and Therapy, 41(5), 509-528. https://doi. org/10.1016/S0005-7967(02)00088-8.

Fairburn, C. G., Cooper, Z., Doll, H. A., O’Connor, M. E., Bohn, K., Hawker, D. M., Wales, J. A., \& Palmer, R. L. (2009). Transdiagnostic cognitive-behavioral therapy for patients with eating disorders: a two-site trial with 60-week follow-up. American Journal of Psychiatry, 166(3), 311-319. https://doi.org/10.1176/ appi.ajp.2008.08040608.

Fernandez, K. C., Fisher, A. J., \& Chi, C. (2017). Development and initial implementation of the Dynamic Assessment Treatment Algorithm (DATA). PLoS One, 12(6), 0178806. https://doi.org/10.1371/journal. pone.0178806.

Fisher, A. J., \& Boswell, J. F. (2016). Enhancing the personalization of psychotherapy with dynamic assessment and modeling. Assessment, 23(4), 496-506. https://doi.org/10.1177/1073191116638735.

Fisher, A. J., Bosley, H. G., Fernandez, K. C., Reeves, J. W., Soyster, P. D., Diamond, A. E., \& Barkin, J. (2019). Open trial of a personalized modular treatment for mood and anxiety. Behaviour research and therapy, 116, 69-79. Behaviour Research and Therapy, 116, 69-79.

Forsell, E., Jernelöv, S., Blom, K., Kraepelien, M., Svanborg, C., Andersson, G., Lindefors, N., \& Kaldo, V. (2019). Proof of concept for an adaptive treatment strategy to prevent failures in internet-delivered CBT: a 
single-blind randomized clinical trial with insomnia patients. American Journal of Psychiatry, 176(4), 315-323. https://doi.org/10.1176/appi.ajp.2018.18060699.

Fusar-Poli, P., Solmi, M., Brondino, N., Davies, C., Chae, C., Politi, P., Borgwardt, S., Lawrie, S. M., Parnas, J., \& McGuire, P. (2019). Transdiagnostic psychiatry: a systematic review. World Psychiatry : Official Journal of the World Psychiatric Association (WPA), 18(2), 192-207. https://doi.org/10.1002/wps.20631.

Goldberg, S. B., Tucker, R. P., Greene, P. A., Davidson, R. J., Wampold, B. E., Kearney, D. J., \& Simpson, T. L. (2018). Mindfulness-based interventions for psychiatric disorders: a systematic review and metaanalysis. Clinical Psychology Review, 59, 52-60. https://doi.org/10.1016/j.cpr.2017.10.011.

González-Robles, A., Díaz-García, A., Miguel, C., García-Palacios, A., \& Botella, C. (2018). Comorbidity and diagnosis distribution in transdiagnostic treatments for emotional disorders: a systematic review of randomized controlled trials. PLoS One, 13(11), 0207396. https://doi.org/10.1371/journal.pone.0207396.

Grawe, K. (2004). Psychological therapy. Boston: Hogrefe Publishing.

Grawe, K., Caspar, F., \& Ambühl, H. (1990). Die Berner Therapievergleichsstudie: Fragestellung und Versuchsplan. Zeitschrift für Klinische Psychologie, 19(4), 294-315.

Grawe, K., Donati, R., \& Bernauer, F. (1994). Psychotherapie im Wandel: Von der Konfession zur Profession (3rd ed.). Göttingen: Hogrefe, Verlag für Psychologie.

Gros, D. F. (2014). Development and initial evaluation of transdiagnostic behavior therapy (TBT) for veterans with affective disorders. Psychiatry Research, 220(1-2), 275-282. https://doi.org/10.1016/j.psychres. 2014.08.018.

Hacker, T., Stone, P., \& MacBeth, A. (2016). Acceptance and commitment therapy - Do we know enough? Cumulative and sequential meta-analyses of randomized controlled trials. Journal of Affective Disorders, 190, 551-565. https://doi.org/10.1016/j.jad.2015.10.053.

Harvey, A. G., Watkins, E. R., Mansell, W., \& Shafran, R. (2004). Cognitive behavioural processes across psychological disorders: a transdiagnostic approach to research and treatment. Oxford: Oxford University Press.

Hayes, S. C., Wilson, K. G., Gifford, E. V., Follette, V. M., \& Strosahl, K. (1996). Experiential avoidance and behavioral disorders: a functional dimensional approach to diagnosis and treatment. Journal of Consulting and Clinical Psychology, 64(6), 1152-1168. https://doi.org/10.1037/0022-006X.64.6.1152.

Hayes, S. C., Strosahl, K. D., \& Wilson, K. G. (1999). Acceptance and commitment therapy: an experiential approach to behavior change. New York: Guilford Press.

Hayes, S. C., Luoma, J. B., Bond, F. W., Masuda, A., \& Lillis, J. (2006). Acceptance and commitment therapy: model, processes and outcomes. Behaviour Research and Therapy, 44(1), 1-25. https://doi.org/ 10.1016/j.brat.2005.06.006.

Hofmann, S. G., \& Hayes, S. C. (2019). The future of intervention science: process-based therapy. Clinical Psychological Science: A Journal of the Association for Psychological Science, 7(1), 37-50. https://doi. org/10.1177/2167702618772296.

Hofmann, S. G., Asnaani, A., Vonk, I. J. J., Sawyer, A. T., \& Fang, A. (2012). The efficacy of cognitive behavioral therapy: a review of meta-analyses. Cognitive Therapy and Research, 36(5), 427-440. https:// doi.org/10.1007/s10608-012-9476-1.

Hong, R. Y., \& Cheung, M. W.-L. (2015). The structure of cognitive vulnerabilities to depression and anxiety. Clinical Psychological Science: A Journal of the Association for Psychological Science, 3(6), 892-912. https://doi.org/10.1177/2167702614553789.

Hopwood, C. J., Bagby, R. M., Gralnick, T., Ro, E., Ruggero, C., Mullins-Sweatt, S., Kotov, R., Bach, B., Cicero, D. C., Krueger, R. F., Patrick, C. J., Chmielewski, M., DeYoung, C. G., Docherty, A. R., Eaton, N. R., Forbush, K. T., Ivanova, M. Y., Latzman, R. D., Pincus, et al. (2019). Integrating psychotherapy with the hierarchical taxonomy of psychopathology (HiTOP). Journal of Psychotherapy Integration https://doi.org/10.1037/int0000156.

Insel, T. R., \& Cuthbert, B. N. (2015). Brain disorders? Precisely. Science, 348(6234), 499-500. https://doi. org/10.1126/science.aab2358.

Insel, T., Cuthbert, B., Garvey, M., Heinssen, R., Pine, D. S., Quinn, K., Sanislow, C., \& Wang, P. (2010). Research domain criteria (RDoC): toward a new classification framework for research on mental disorders. American Journal of Psychiatry, 167(7), 748-751. https://doi.org/10.1176/appi.ajp.2010. 09091379.

Jacob, G. A., \& Arntz, A. (2013). Schema therapy for personality disorders - a review. International Journal of Cognitive Therapy, 6(2), 171-185. https://doi.org/10.1521/ijct.2013.6.2.171.

Jacobi, F., Höfler, M., Siegert, J., Mack, S., Gerschler, A., Scholl, L., Busch, M. A., Hapke, U., Maske, U., Seiffert, I., Gaebel, W., Maier, W., Wagner, M., Zielasek, J., \& Wittchen, H.-U. (2014). Twelve-month prevalence, comorbidity and correlates of mental disorders in Germany: The mental health module of the 
German Health Interview and Examination Survey for Adults (DEGS1-MH). International Journal of Methods in Psychiatric Research, 23(3), 304-319. https://doi.org/10.1002/mpr.1439.

Johansson, R., Sjöberg, E., Sjögren, M., Johnsson, E., Carlbring, P., Andersson, T., Rousseau, A., \& Andersson, G. (2012). Tailored vs. standardized internet-based cognitive behavior therapy for depression and comorbid symptoms: a randomized controlled trial. PloS One, 7(5), 36905. https://doi.org/10.1371/ journal.pone.0036905.

Kabat-Zinn, J. (2003). Mindfulness-based interventions in context: past, present, and future. Clinical Psychology: Science and Practice, 10(2), 144-156. https://doi.org/10.1093/clipsy/bpg016.

Käll, A., Jägholm, S., Hesser, H., Andersson, F., Mathaldi, A., Norkvist, B. T., Shafran, R., \& Andersson, G. (2020). Internet-based cognitive behavior therapy for loneliness: a pilot randomized controlled trial. Behavior Therapy, 51(1), 54-68. https://doi.org/10.1016/j.beth.2019.05.001.

Kotov, R., Krueger, R. F., Watson, D., Achenbach, T. M., Althoff, R. R., Bagby, R. M., Brown, T. A., Carpenter, W. T., Caspi, A., Clark, L. A., Eaton, N. R., Forbes, M. K., Forbush, K. T., Goldberg, D., Hasin, D., Hyman, S. E., Ivanova, M. Y., Lynam, D. R., Markon, K., et al. (2017). The Hierarchical Taxonomy of Psychopathology (HiTOP): a dimensional alternative to traditional nosologies. Journal of Abnormal Psychology, 126(4), 454-477. https://doi.org/10.1037/abn0000258.

Kraepelien, M., Svanborg, C., Lallerstedt, L., Sennerstam, V., Lindefors, N., \& Kaldo, V. (2019). Individually tailored internet treatment in routine care: a feasibility study. Internet Interventions, 18, 100263. https:// doi.org/10.1016/j.invent.2019.100263.

Kramer, U., Kolly, S., Berthoud, L., Keller, S., Preisig, M., Caspar, F., Berger, T., de Roten, Y., Marquet, P., \& Despland, J.-N. (2014). Effects of motive-oriented therapeutic relationship in a ten-session general psychiatric treatment of borderline personality disorder: a randomized controlled trial. Psychotherapy and Psychosomatics, 83(3), 176-186. https://doi.org/10.1159/000358528.

Lahey, B. B., Krueger, R. F., Rathouz, P. J., Waldman, I. D., \& Zald, D. H. (2017a). Validity and utility of the general factor of psychopathology. World Psychiatry, 16(2), 142-144. https://doi.org/10.1002/wps. 20410.

Lahey, B. B., Krueger, R. F., Rathouz, P. J., Waldman, I. D., \& Zald, D. H. (2017b). A hierarchical causal taxonomy of psychopathology across the life span. Psychological Bulletin, 143(2), 142-186. https://doi. org/10.1037/bu10000069.

Lambert, M. J., \& Shimokawa, K. (2011). Collecting client feedback. Psychotherapy, 48(1), 72-79. https:// doi.org/10.1037/a0022238.

Layard, R., \& Clark, D. M. (2014). Thrive: how better mental health care transforms lives and saves money. Princeton University Press. https://doi.org/10.1515/9781400873333.

Leichsenring, F., \& Salzer, S. (2014). A unified protocol for the transdiagnostic psychodynamic treatment of anxiety disorders: an evidence-based approach. Psychotherapy, 51(2), 224-245. https://doi.org/10.1037/ a0033815.

Leichsenring, F., \& Schauenburg, H. (2014). Empirically supported methods of short-term psychodynamic therapy in depression - Towards an evidence-based unified protocol. Journal of Affective Disorders, 169, 128-143. https://doi.org/10.1016/j.jad.2014.08.007.

Leichsenring, F., \& Steinert, C. (2018). Towards an evidence-based unified psychodynamic protocol for emotional disorders. Journal of Affective Disorders, 232, 400-416. https://doi.org/10.1016/j.jad.2017.11. 036.

Leichsenring, F., Salzer, S., Jaeger, U., Kachele, H., Kreische, R., Leweke, F., Ruger, U., Winkelbach, C., \& Leibing, E. (2009). Short-term psychodynamic psychotherapy and cognitive-behavioral therapy in generalized anxiety disorder: a randomized, controlled trial. American Journal of Psychiatry, 166(8), 875-881. https://doi.org/10.1176/appi.ajp.2009.09030441.

Leichsenring, F., Salzer, S., Beutel, M. E., Herpertz, S., Hiller, W., Hoyer, J., Huesing, J., Joraschky, P., Nolting, B., Poehlmann, K., Ritter, V., Stangier, U., Strauss, B., Stuhldreher, N., Tefikow, S., Teismann, T., Willutzki, U., Wiltink, J., \& Leibing, E. (2013). Psychodynamic therapy and cognitive-behavioral therapy in social anxiety disorder: a multicenter randomized controlled trial. American Journal of Psychiatry, 170(7), 759-767. https://doi.org/10.1176/appi.ajp.2013.12081125.

Linehan, M. (1993a). Skills training manual for treating borderline personality disorder. New York: Guilford Press.

Linehan, M. (1993b). Cognitive-behavioral treatment of borderline personality disorder. New York: Guilford Press.

Lungu, A., \& Linehan, M. M. (2016). Dialectical behavior therapy: a comprehensive multi-and transdiagnostic intervention. In C. M. Nezu \& A. M. Nezu (Eds.), The Oxford handbook of cognitive and behavioral therapies (pp. 200-214). Oxford: Oxford University Press. 
MacLeod, C., \& Mathews, A. (2012). Cognitive bias modification approaches to anxiety. Annual Review of Clinical Psychology, 8(1), 189-217. https://doi.org/10.1146/annurev-clinpsy-032511-143052.

Mahoney, A. E. J., \& McEvoy, P. M. (2012). A transdiagnostic examination of intolerance of uncertainty across anxiety and depressive disorders. Cognitive Behaviour Therapy, 41(3), 212-222. https://doi.org/ 10.1080/16506073.2011.622130.

Maia, A. C. C. d. O., Braga, A. A., Nunes, C. A., Nardi, A. E., \& Silva, A. C. (2013). Transdiagnostic treatment using a unified protocol: application for patients with a range of comorbid mood and anxiety disorders. Trends in Psychiatry and Psychotherapy, 35(2), 134-140. https://doi.org/10.1590/S223760892013000200007.

Mansell, W., \& McEvoy, P. M. (2017). A test of the core process account of psychopathology in a heterogenous clinical sample of anxiety and depression. A case of the blind men and the elephant? Journal of Anxiety Disorders, 46, 4-10.

Mansell, W., Harvey, A., Watkins, E. R., \& Shafran, R. (2008). Cognitive behavioral processes across psychological disorders: a review of the utility and validity of the transdiagnostic approach. International Journal of Cognitive Therapy, 1(3), 181-191. https://doi.org/10.1521/ijct.2008.1.3.181.

Mansell, W., Harvey, A., Watkins, E., \& Shafran, R. (2009). Conceptual foundations of the transdiagnostic approach to CBT. Journal of Cognitive Psychotherapy, 23(1), 6-19. https://doi.org/10.1891/0889-8391. 23.1.6.

Martell, C. R., Dimidjian, S., \& Herman-Dunn, R. (2013). Behavioral activation for depression: a clinician's guide. New York: Guilford Press.

McEvoy, P. M., \& Erceg-Hurn, D. M. (2016). The search for universal transdiagnostic and trans-therapy change processes: evidence for intolerance of uncertainty. Journal of Anxiety Disorders, 41, 96-107. https://doi.org/10.1016/j.janxdis.2016.02.002.

McEvoy, P. M., Nathan, P., \& Norton, P. J. (2009). Efficacy of transdiagnostic treatments: a review of published outcome studies and future research directions. Journal of Cognitive Psychotherapy, 23(1), 2033. https://doi.org/10.1891/0889-8391.23.1.20.

Mennin, D. S., \& Fresco, D. M. (2014). Emotion regulation therapy. In J. J. Gross (Ed.), Handbook of Emotion Regulation (2nd ed., pp. 469-490). New York: Guilford Press.

Morris, L., \& Mansell, W. (2018). A systematic review of the relationship between rigidity/flexibility and transdiagnostic cognitive and behavioral processes that maintain psychopathology. Journal of Experimental Psychopathology, 9(3), 204380871877943. https://doi.org/10.1177/2043808718779431.

Murray, L. K., Dorsey, S., Haroz, E., Lee, C., Alsiary, M. M., Haydary, A., Weiss, W. M., \& Bolton, P. (2014). A common elements treatment approach for adult mental health problems in low- and middleincome countries. Cognitive and Behavioral Practice, 21(2), 111-123. https://doi.org/10.1016/j.cbpra. 2013.06.005.

Naragon-Gainey, K., \& Watson, D. (2018). What lies beyond neuroticism? An examination of the unique contributions of social-cognitive vulnerabilities to internalizing disorders. Assessment, 25(2), 143-158. https://doi.org/10.1177/1073191116659741.

Naragon-Gainey, K., McMahon, T. P., \& Chacko, T. P. (2017). The structure of common emotion regulation strategies: a meta-analytic examination. Psychological Bulletin, 143(4), 384-427. https://doi.org/10.1037/ bul0000093.

Neacsiu, A. D., Eberle, J. W., Kramer, R., Wiesmann, T., \& Linehan, M. M. (2014). Dialectical behavior therapy skills for transdiagnostic emotion dysregulation: a pilot randomized controlled trial. Behaviour Research and Therapy, 59, 40-51. https://doi.org/10.1016/j.brat.2014.05.005.

Newby, J. M., Mackenzie, A., Williams, A. D., McIntyre, K., Watts, S., Wong, N., \& Andrews, G. (2013). Internet cognitive behavioural therapy for mixed anxiety and depression: a randomized controlled trial and evidence of effectiveness in primary care. Psychological Medicine, 43(12), 2635-2648. https://doi. org/10.1017/S0033291713000111.

Newby, J. M., McKinnon, A., Kuyken, W., Gilbody, S., \& Dalgleish, T. (2015). Systematic review and metaanalysis of transdiagnostic psychological treatments for anxiety and depressive disorders in adulthood. Clinical Psychology Review, 40, 91-110. https://doi.org/10.1016/j.cpr.2015.06.002.

Newby, J. M., Twomey, C., Yuan Li, S. S., \& Andrews, G. (2016). Transdiagnostic computerised cognitive behavioural therapy for depression and anxiety: a systematic review and meta-analysis. Journal of Affective Disorders, 199, 30-41. https://doi.org/10.1016/j.jad.2016.03.018.

Norcross, J. C., \& Wampold, B. E. (2018). A new therapy for each patient: evidence-based relationships and responsiveness. Journal of Clinical Psychology, 74(11), 1889-1906. https://doi.org/10.1002/jclp.22678.

Nordgren, L. B., Andersson, G., Kadowaki, Å., \& Carlbring, P. (2012). Tailored internet-administered treatment of anxiety disorders for primary care patients: study protocol for a randomised controlled trial. Trials, 13(1), 16. https://doi.org/10.1186/1745-6215-13-16. 
Norton, P. J. (2012). A randomized clinical trial of transdiagnostic cognitve-behavioral treatments for anxiety disorder by comparison to relaxation training. Behavior Therapy, 43(3), 506-517. https://doi.org/10.1016/ j.beth.2010.08.011.

Norton, P. J., \& Barrera, T. L. (2012). Transdiagnostic versus diagnosis-specific CBT for anxiety disorders: a preliminary randomized controlled noninferiority trial. Depression and Anxiety, 29(10), 874-882. https:// doi.org/10.1002/da.21974.

Norton, P. J., \& Hope, D. A. (2005). Preliminary evaluation of a broad-spectrum cognitive-behavioral group therapy for anxiety. Journal of Behavior Therapy and Experimental Psychiatry, 36(2), 79-97. https://doi. org/10.1016/j.jbtep.2004.07.002.

Ost, L.-G. (2008). Efficacy of the third wave of behavioral therapies: a systematic review and meta-analysis. Behaviour Research and Therapy, 46(3), 296-321. https://doi.org/10.1016/j.brat.2007.12.005.

Ost, L.-G. (2014). The efficacy of acceptance and commitment therapy: an updated systematic review and meta-analysis. Behaviour Research and Therapy, 61, 105-121. https://doi.org/10.1016/j.brat.2014.07. 018.

Panos, P. T., Jackson, J. W., Hasan, O., \& Panos, A. (2014). Meta-analysis and systematic review assessing the efficacy of dialectical behavior therapy (DBT). Research on Social Work Practice, 24(2), 213-223. https://doi.org/10.1177/1049731513503047.

Păsărelu, C. R., Andersson, G., Bergman Nordgren, L., \& Dobrean, A. (2017). Internet-delivered transdiagnostic and tailored cognitive behavioral therapy for anxiety and depression: a systematic review and meta-analysis of randomized controlled trials. Cognitive Behaviour Therapy, 46(1), 1-28. https://doi. org/10.1080/16506073.2016.1231219.

Pearl, S. B., \& Norton, P. J. (2017). Transdiagnostic versus diagnosis specific cognitive behavioural therapies for anxiety: a meta-analysis. Journal of Anxiety Disorders, 46, 11-24. https://doi.org/10.1016/j.janxdis. 2016.07.004.

Persons, J. B. (2012). The case formulation approach to cognitive-behavior therapy. New York: Guilford Press.

Persons, J. B. (2016). Science in practice in cognitive behavior therapy. Cognitive and Behavioral Practice, 23(4), 454 458. https://doi.org/10.1016/j.cbpra.2016.01.003.

Philipp, R., Kriston, L., Lanio, J., Kühne, F., Härter, M., Moritz, S., \& Meister, R. (2019). Effectiveness of metacognitive interventions for mental disorders in adults - a systematic review and meta-analysis (METACOG). Clinical Psychology \& Psychotherapy, 26(2), 227-240. https://doi.org/10.1002/cpp.2345.

Pinquart, M., Oslejsek, B., \& Teubert, D. (2016). Efficacy of systemic therapy on adults with mental disorders: a meta-analysis. Psychotherapy Research, 26(2), 241-257. https://doi.org/10.1080/10503307.2014. 935830.

Reinholt, N., \& Krogh, J. (2014). Efficacy of transdiagnostic cognitive behaviour therapy for anxiety disorders: a systematic review and meta-analysis of published outcome studies. Cognitive Behaviour Therapy, 43(3), 171-184. https://doi.org/10.1080/16506073.2014.897367.

Reinholt, N., Aharoni, R., Winding, C., Rosenberg, N., Rosenbaum, B., \& Arnfred, S. (2017). Transdiagnostic group CBT for anxiety disorders: the unified protocol in mental health services. Cognitive Behaviour Therapy, 46(1), 29-43. https://doi.org/10.1080/16506073.2016.1227360.

Renna, M. E., Quintero, J. M., Fresco, D. M., \& Mennin, D. S. (2017). Emotion regulation therapy: a mechanism-targeted treatment for disorders of distress. Frontiers in Psychology, 8(98), 1-14. https://doi. org/10.3389/fpsyg.2017.00098.

Riccardi, C. J., Korte, K. J., \& Schmidt, N. B. (2017). False safety behavior elimination therapy: a randomized study of a brief individual transdiagnostic treatment for anxiety disorders. Journal of Anxiety Disorders, 46, 35-45. https://doi.org/10.1016/j.janxdis.2016.06.003.

Riedinger, V., Pinquart, M., \& Teubert, D. (2017). Effects of systemic therapy on mental health of children and adolescents: a meta-analysis. Journal of Clinical Child and Adolescent Psychology, 46(6), 880-894. https://doi.org/10.1080/15374416.2015.1063427.

Riley, C., Lee, M., Cooper, Z., Fairburn, C. G., \& Shafran, R. (2007). A randomised controlled trial of cognitive-behaviour therapy for clinical perfectionism: a preliminary study. Behaviour Research and Therapy, 45(9), 2221-2231. https://doi.org/10.1016/j.brat.2006.12.003.

Ritschel, L. A., Lim, N. E., \& Stewart, L. M. (2015). Transdiagnostic applications of DBT for adolescents and adults. American Journal of Psychotherapy, 69(2), 111-128.

Sakiris, N., \& Berle, D. (2019). A systematic review and meta-analysis of the unified protocol as a transdiagnostic emotion regulation based intervention. Clinical Psychology Review, 72, 101751. https:// doi.org/10.1016/j.cpr.2019.101751. 
Sauer-Zavala, S., Gutner, C. A., Farchione, T. J., Boettcher, H. T., Bullis, J. R., \& Barlow, D. H. (2017). Current definitions of "transdiagnostic" in treatment development: a search for consensus. Behavior Therapy, 48, 128-138. https://doi.org/10.1016/j.beth.2016.09.004.

Sauer-Zavala, S., Cassiello-Robbins, C., Ametaj, A. A., Wilner, J. G., \& Pagan, D. (2019). Transdiagnostic treatment personalization: the feasibility of ordering unified protocol modules according to patient strengths and weaknesses. Behavior Modification, 43(4), 518-543. https://doi.org/10.1177/ 0145445518774914.

Schmidt, N. B., Buckner, J. D., Pusser, A., Woolaway-Bickel, K., Preston, J. L., \& Norr, A. (2012). Randomized controlled trial of false safety behavior elimination therapy: a unified cognitive behavioral treatment for anxiety psychopathology. Behavior Therapy, 43(3), 518-532. https://doi.org/10.1016/j.beth. 2012.02.004.

Segal, Z. V., Williams, J. M. G., \& Teasdale, J. D. (2002). Mindfulness-based cognitive therapy for depression: a new approach to preventing relapse. New York: Guilford Press.

Shackman, A. J., Tromp, D. P. M., Stockbridge, M. D., Kaplan, C. M., Tillman, R. M., \& Fox, A. S. (2016). Dispositional negativity: an integrative psychological and neurobiological perspective. Psychological Bulletin, 142(12), 1275-1314. https://doi.org/10.1037/bul0000073.

Shafran, R., Lee, M., \& Fairburn, C. G. (2004). Clinical perfectionism: a case report. Behavioural and Cognitive Psychotherapy, 32(3), 353-357. https://doi.org/10.1017/S1352465804001420.

Shihata, S., McEvoy, P. M., Mullan, B. A., \& Carleton, R. N. (2016). Intolerance of uncertainty in emotional disorders: what uncertainties remain? Journal of Anxiety Disorders, 41, 115-124. https://doi.org/10.1016/ j.janxdis.2016.05.001.

Silfvernagel, K., Carlbring, P., Kabo, J., Edström, S., Eriksson, J., Månson, L., \& Andersson, G. (2012). Individually tailored internet-based treatment for young adults and adults with panic attacks: randomized controlled trial. Journal of Medical Internet Research, 14(3), 65. https://doi.org/10.2196/jmir.1853.

Silfvernagel, K., Westlinder, A., Andersson, S., Bergman, K., Diaz Hernandez, R., Fallhagen, L., Lundqvist, I., Masri, N., Viberg, L., Forsberg, M.-L., Lind, M., Berger, T., Carlbring, P., \& Andersson, G. (2018). Individually tailored internet-based cognitive behaviour therapy for older adults with anxiety and depression: a randomised controlled trial. Cognitive Behaviour Therapy, 47(4), 286-300. https://doi. org/10.1080/16506073.2017.1388276.

Sloan, E., Hall, K., Moulding, R., Bryce, S., Mildred, H., \& Staiger, P. K. (2017). Emotion regulation as a transdiagnostic treatment construct across anxiety, depression, substance, eating and borderline personality disorders: a systematic review. Clinical Psychology Review, 57, 141-163. https://doi.org/10.1016/j. cpr.2017.09.002.

Smith, E. C., \& Grawe, K. (2005). Which therapeutic mechanisms work when? A step towards the formulation of empirically validated guidelines for therapists' session-to-session decisions. Clinical Psychology \& Psychotherapy, 12(2), 112-123. https://doi.org/10.1002/cpp.427.

Stratton, P. (2016). The evidence base of family therapy and systemic practice. UK: Association for Family Therapy.

Timulak, L., \& Keogh, D. (2020). Emotion-focused therapy: a transdiagnostic formulation. Journal of Contemporary Psychotherapy, 50(1), 1-13. https://doi.org/10.1007/s10879-019-09426-7.

Titov, N., Andrews, G., Johnston, L., Robinson, E., \& Spence, J. (2010). Transdiagnostic internet treatment for anxiety disorders: a randomized controlled trial. Behaviour Research and Therapy, 48(9), 890-899. https://doi.org/10.1016/j.brat.2010.05.014.

Titov, N., Dear, B. F., Schwencke, G., Andrews, G., Johnston, L., Craske, M. G., \& McEvoy, P. (2011). Transdiagnostic internet treatment for anxiety and depression: a randomised controlled trial. Behaviour Research and Therapy, 49(8), 441-452. https://doi.org/10.1016/j.brat.2011.03.007.

Tulbure, B. T., Rusu, A., Sava, F. A., Sălăgean, N., \& Farchione, T. J. (2018). A web-based transdiagnostic intervention for affective and mood disorders: randomized controlled trial. JMIR Mental Health, 5(2), 36. https://doi.org/10.2196/mental.8901.

Watkins, E. R., Scott, J., Wingrove, J., Rimes, K., Bathurst, N., Steiner, H., Kennell-Webb, S., Moulds, M., \& Malliaris, Y. (2007). Rumination-focused cognitive behaviour therapy for residual depression: a case series. Behaviour Research and Therapy, 45(9), 2144-2154. https://doi.org/10.1016/j.brat.2006.09.018.

Watkins, E. R., Mullan, E., Wingrove, J., Rimes, K., Steiner, H., Bathurst, N., Eastman, R., \& Scott, J. (2011). Rumination-focused cognitive-behavioural therapy for residual depression: phase II randomised controlled trial. The British Journal of Psychiatry, 199(4), 317-322. https://doi.org/10.1192/bjp.bp.110. 090282.

Weiss, W. M., Murray, L. K., Zangana, G. A. S., Mahmooth, Z., Kaysen, D., Dorsey, S., Lindgren, K., Gross, A., Murray, S. M., Bass, J. K., \& Bolton, P. (2015). Community-based mental health treatments for 
survivors of torture and militant attacks in southern Iraq: a randomized control trial. BMC Psychiatry, 15(1), 249. https://doi.org/10.1186/s12888-015-0622-7.

Weisz, J. R., Chorpita, B. F., Palinkas, L. A., Schoenwald, S. K., Miranda, J., Bearman, S. K., Daleiden, E. L., Ugueto, A. M., Ho, A., Martin, J., Gray, J., Alleyne, A., Langer, D. A., Southam-Gerow, M. A., Gibbons, R. D., \& the Research Network on Youth Mental Health. (2012). Testing standard and modular designs for psychotherapy treating depression, anxiety, and conduct problems in youth: a randomized effectiveness trial. Archives of General Psychiatry, 69(3), 274-282. https://doi.org/10.1001/archgenpsychiatry. 2011.147.

Weisz, J. R., Ng, M. Y., \& Bearman, S. K. (2014). Odd couple? Reenvisioning the relation between science and practice in the dissemination-implementation era. Clinical Psychological Science: A Journal of the Association for Psychological Science, 2(1), 58-74. https://doi.org/10.1177/2167702613501307.

Wells, A. (2011). Metacognitive therapy for anxiety and depression. New York: Guilford Press.

Wright, A. G. C., \& Woods, W. C. (2020). Personalized models of psychopathology. Annual Review of Clinical Psychology, 16(1), 49-74. https://doi.org/10.1146/annurev-clinpsy-102419-125032.

Young, J. E. (1999). Cognitive therapy for personality disorders: A schema-focused approach, 3rd ed (3rd ed.). Sarasota: Professional Resource Press.

Publisher's Note Springer Nature remains neutral with regard to jurisdictional claims in published maps and institutional affiliations. 